\title{
Observational study of talimogene laherparepvec use for melanoma in clinical practice in the United States (COSMUS-1)
}

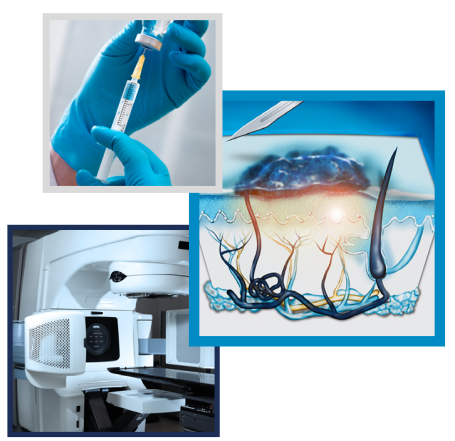

\author{
Matthew C Perez ${ }^{\ddagger 1}$, Jonathan S Zager*, ${ }^{\star 1}$, Thomas Amatruda², Robert Conry ${ }^{3}$, Charlotte \\ ${ }^{1}$ Moffitt Cancer Center, Tampa, FL 33612, USA \\ ${ }^{2}$ Minnesota Oncology, Fridley, MN 55432, USA \\ ${ }^{3}$ The University of Alabama at Birmingham, Birmingham, AL 35294, USA \\ ${ }^{4}$ Memorial Sloan Kettering Cancer Center, NY 10065, USA \\ ${ }^{5}$ Beth Israel Deaconess Medical Center, Boston, MA 02215, USA \\ ${ }^{6}$ UPMC Hillman Center, Department of Medicine, University of Pittsburgh, Pittsburgh, PA 15232, USA \\ ${ }^{7}$ Amgen Inc., Thousand Oaks, CA 91320, USA \\ *Author for correspondence: Tel.: + 1813361 0956; jonathan.zager@moffitt.org \\ $\ddagger$ Authors contributed equally
} Ariyan $^{4}$, Anupam Desai ${ }^{5}$, John M Kirkwood ${ }^{6}$, Sheryl Treichell, David Cohan ${ }^{7}$ \& Leon Raskin ${ }^{7}$

\section{Summary points}

- Talimogene laherparepvec (T-VEC) is an intralesional oncolytic viral therapy and the first oncolytic viral therapy approved by the US FDA.

- T-VEC was the first oncolytic immunotherapy to be approved in Europe and was approved for adults with unresectable stage IIIB, IIIC or IVM1a melanoma.

- According to this medical chart review conducted at seven academic centers and specialty clinics in the US, T-VEC was well tolerated and showed clinical utility in 76 patients with unresectable metastatic melanoma.

- This real-world cohort was older and more heavily pretreated than the patient population in the pivotal trial for T-VEC.

- A substantial proportion of patients (43.4\%) received checkpoint inhibitors before or concurrent with T-VEC.

- Twenty percent of patients completed treatment with achievement of pathological complete response or no remaining injectable lesions.

- Flu-like symptoms and injection site complications occurred at rates similar to those observed in other recent studies.

- No herpetic lesions or infections were reported.

Aim: Talimogene laherparepvec (T-VEC) is an intralesional treatment for unresectable cutaneous, subcutaneous and nodal melanoma. COSMUS-1 was conducted to examine how T-VEC is used in US clinical practice. Materials \& methods: A chart review was conducted at seven centers, with 78 patients screened and 76 eligible. Results: Patients began treatment with T-VEC between October 2015 and December 2016. Median follow-up was 9.4 months. Twenty percent of patients $(n=15)$ completed T-VEC treatment with no remaining injectable lesions or pathologic complete response. Flu-like symptoms were the most commonly reported adverse events $(n=8 ; 10.5 \%)$, followed by lesion ulceration $(n=4 ; 5.3 \%)$. No herpetic lesions or infections were reported. Conclusion: T-VEC was well tolerated and showed clinical utility.

First draft submitted: 4 April 2019; Accepted for publication: 17 June 2019; Published online:

3 July 2019

Keywords: combination therapy • immunotherapy • melanoma • metastatic melanoma $\bullet$ oncolytic virus $\bullet$ real-world evidence $\bullet$ T-VEC • TVEC • talimogene laherparepvec

Although there have been major advances in the treatment of advanced melanoma in the last decade [1,2], the prognosis for patients with melanoma still depends on the disease stage at presentation [3], and treatment of locally advanced and metastatic disease still pose significant challenges $[4,5]$. 


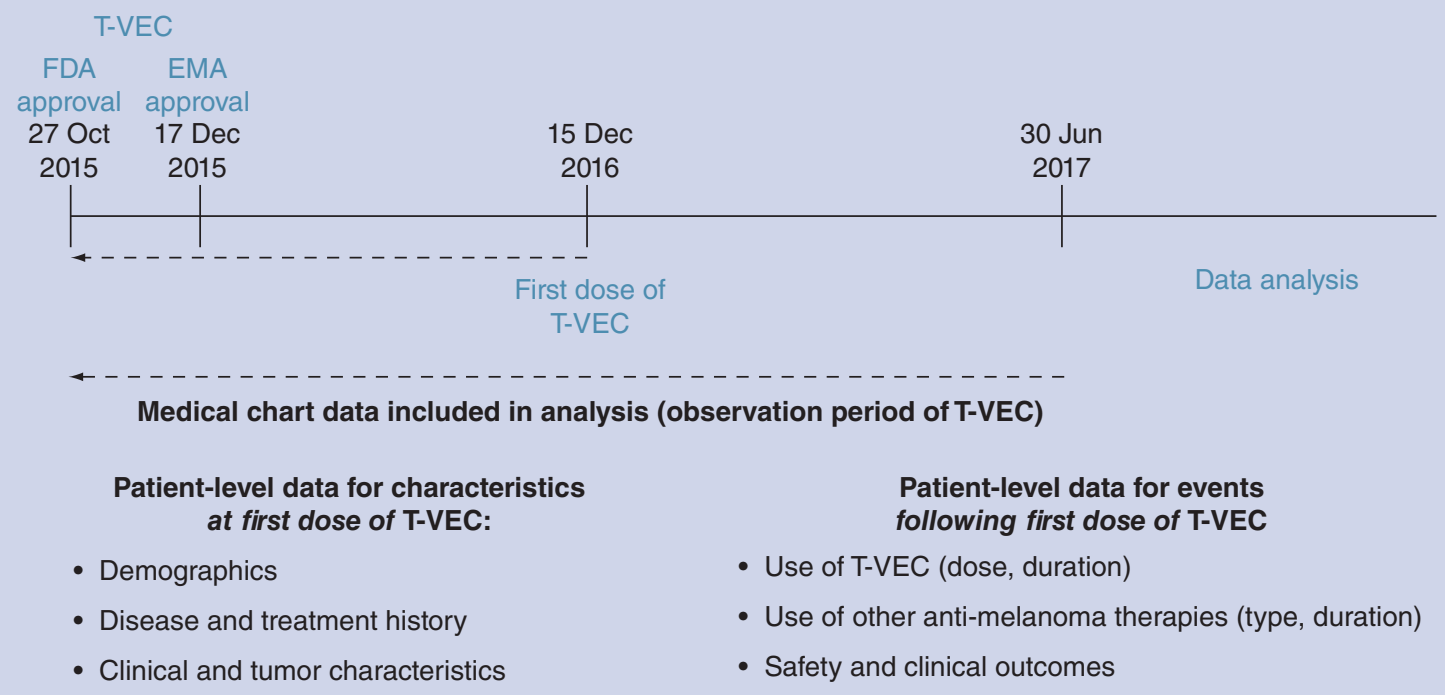

Figure 1. Study design schema for COSMUS-1.

EMA: European Medicines Agency; FDA: Food and Drug Administration; T-VEC: Talimogene laherparepvec.

Talimogene laherparepvec (T-VEC) is an intralesional oncolytic viral therapy and the first oncolytic viral therapy approved by the US FDA [6]. The US approval was based on data from the Phase III Oncovex (granulocyte macrophage colony-stimulating factor [GM-CSF]) Pivotal Trial in Melanoma (OPTiM) trial in stage IIIB-IVM1c melanoma [7]. T-VEC was also the first oncolytic immunotherapy to be approved in Europe, where approval was for adults with unresectable stage IIIB, IIIC or IVM1a melanoma [8]. The OPTiM study, when analyzed by stage of disease, showed that treatment with T-VEC significantly improved overall survival (OS) in patients with stage IIIB, IIIC and IVM1a melanoma, but not stage IVM1b or M1c, when compared with an alternative immunologic intervention [7].

T-VEC is an oncolytic herpes simplex virus type 1 (HSV-1). HSV-1 is particularly appealing as an oncolytic viral platform due to its lytic lifecycle, its large genome $(152 \mathrm{~kb})$ amenable to genetic payloads and its well-characterized biology [9]. To produce T-VEC, HSV-1 is modified by the deletion of a neurovirulence gene (ICP34.5) and an immunogenicity gene (ICP47) and the addition of the two gene copies encoding human GM-CSF [9]. These modifications stimulate viral pathogenicity, enhance tumor-selective replication, reduce virally mediated suppression of antigen presentation and induce tumor-specific T-cell responses [9-11].

The OPTiM trial completed enrollment in 2011 [7]. Since that time, there have been other major practicechanging shifts in the treatment landscape for advanced melanoma. The use of immunotherapy (ipilimumab, nivolumab, pembrolizumab) and targeted therapy (vemurafenib, dabrafenib, trametinib, cobimetinib, binimetinib, encorafenib) is now routine, resulting in improved OS in patients with advanced melanoma [4]. Given these recent dramatic changes, the COSMUS-1 study was proposed to assess the use of T-VEC in treatment of advanced melanoma in routine clinical practice in the US.

\section{Materials \& methods}

COSMUS-1 was a case series in which data were gathered from medical chart review of patients treated with T-VEC from 2016 to 2017 at seven academic centers and specialty clinics in the US. The objectives were to describe the clinical characteristics of patients at first dose of T-VEC, use of T-VEC (volume, duration), use of other antimelanoma therapies, clinical outcomes and safety (Figure 1).

To be eligible, patients must have started treatment with T-VEC between 27 October 2015 and 15 December 2016. Patients who participated in a clinical trial or an expanded access program for T-VEC were excluded. Medical chart data were transcribed into an electronic case report form for analysis.

Analyses characterized patient demographics (sex and age) and disease history (date of first diagnosis, stage at diagnosis, and types and dates of metastases). Clinical characteristics (Eastern Cooperative Oncology Group 
Table 1. Baseline demographics and clinical characteristics.

\begin{tabular}{|c|c|c|c|c|}
\hline Baseline characteristic & Stage IIIB-IVM1a $(n=42)$ & Stage IVM1b-IVM1c $(n=30)$ & Stage unknown $(n=4)$ & Total $(n=76)$ \\
\hline Sex (male), n (\%) & $23(54.8)$ & $21(70.0)$ & $1(25.0)$ & $45(59.2)$ \\
\hline \multicolumn{5}{|l|}{ Age, $n(\%)$ : } \\
\hline$-<65$ years & $13(31.0)$ & $10(33.3)$ & $1(25.0)$ & $24(31.6)$ \\
\hline$-65-74$ years & $6(14.3)$ & $11(36.7)$ & $1(25.0)$ & $18(23.7)$ \\
\hline$-\geq 75$ years & $23(54.8)$ & $9(30.0)$ & $2(50.0)$ & $34(44.7)$ \\
\hline \multicolumn{5}{|c|}{ ECOG performance status, $n(\%)$ : } \\
\hline-1 & $11(26.2)$ & $13(43.3)$ & $2(50.0)$ & $26(34.2)$ \\
\hline-2 & $0(0.0)$ & $4(13.3)$ & $0(0.0)$ & $4(5.3)$ \\
\hline-3 & $0(0.0)$ & $2(6.7)$ & $0(0.0)$ & $2(2.6)$ \\
\hline - Unknown & $21(50.0)$ & $3(10.0)$ & $0(0.0)$ & $24(31.6)$ \\
\hline \multicolumn{5}{|c|}{ BRAF mutation status, $\mathrm{n}(\%)$ : } \\
\hline$-\leq 1$ ULN & $18(42.9)$ & $9(30.0)$ & $2(50.0)$ & $29(38.2)$ \\
\hline$->1$ ULN & $5(11.9)$ & $5(16.7)$ & $1(25.0)$ & $11(14.5)$ \\
\hline$->1$ ULN $\leq 2$ ULN & $5(11.9)$ & $3(10.0)$ & $1(25.0)$ & $9(11.8)$ \\
\hline$->2$ ULN & $0(0.0)$ & $2(6.7)$ & $0(0.0)$ & $2(2.6)$ \\
\hline - Unknown & $19(45.2)$ & $16(53.3)$ & $1(25.0)$ & $36(47.4)$ \\
\hline
\end{tabular}

performance status [ECOG-PS], serum LDH level, and BRAF mutation status) and tumor characteristics (presence, number, and anatomical regions of cutaneous lesions, subcutaneous lesions and nodes at first dose of T-VEC) were also recorded.

The patterns of use of T-VEC and other melanoma therapies (type, duration) were analyzed as were safety and clinical outcomes (reason for treatment discontinuation, survival and adverse events [AEs] of interest, including herpetic events). In addition, the use of prior, subsequent or concurrent checkpoint inhibitors was examined.

\section{Results}

\section{Patient characteristics}

The medical records of 78 patients were screened, of whom 76 were eligible and were included in the analysis (two were not included due to participation in a T-VEC clinical trial or expanded access program). The median age of included patients was 73 years (range, 30-93 years); 59.2\% ( $\mathrm{n}=45)$ were male. Table 1 describes patient demographics in detail and Figure 2 shows the disposition of patients.

The median duration of follow-up was 9.4 months (range, 0.1-17.0 months), and eight patients (10.5\%) remained on T-VEC at the end of data collection. Patients received a median of six doses of T-VEC (range, 1-19 doses). American Joint Committee on Cancer 7th edition staging criteria were used [12]. At the time of the first dose, 42 patients (55.3\%) had stage IIIB-IVM1a melanoma, 30 patients (39.5\%) had stage IVM1b-IVM1c disease and in four patients $(5.3 \%)$ the stage of disease was unknown. Cutaneous lesions were present in 41 patients $(53.9 \%)$, subcutaneous lesions were present in 43 patients $(56.6 \%)$ and 25 patients $(32.9 \%)$ had injectable nodal disease. Distant metastases were diagnosed prior to the first dose of T-VEC in 29 patients (38.2\%).

\section{Overall survival}

The median follow-up of the cohort was 9.4 months at the end of the data collection. OS rates at 1 year were $76.7 \%$ in patients with stage IIIB-IVM1a melanoma $(\mathrm{n}=42)$ and $64.6 \%$ in patients with stage IVM1b-IVM1c disease $(\mathrm{n}=30)$. Twenty patients $(26.3 \%)$ died. Thirteen of these deaths were due to disease progression $(65.0 \%)$, two $(10.0 \%)$ were due to co-morbidities, and for five $(25.0 \%)$ the cause of death was not reported. 


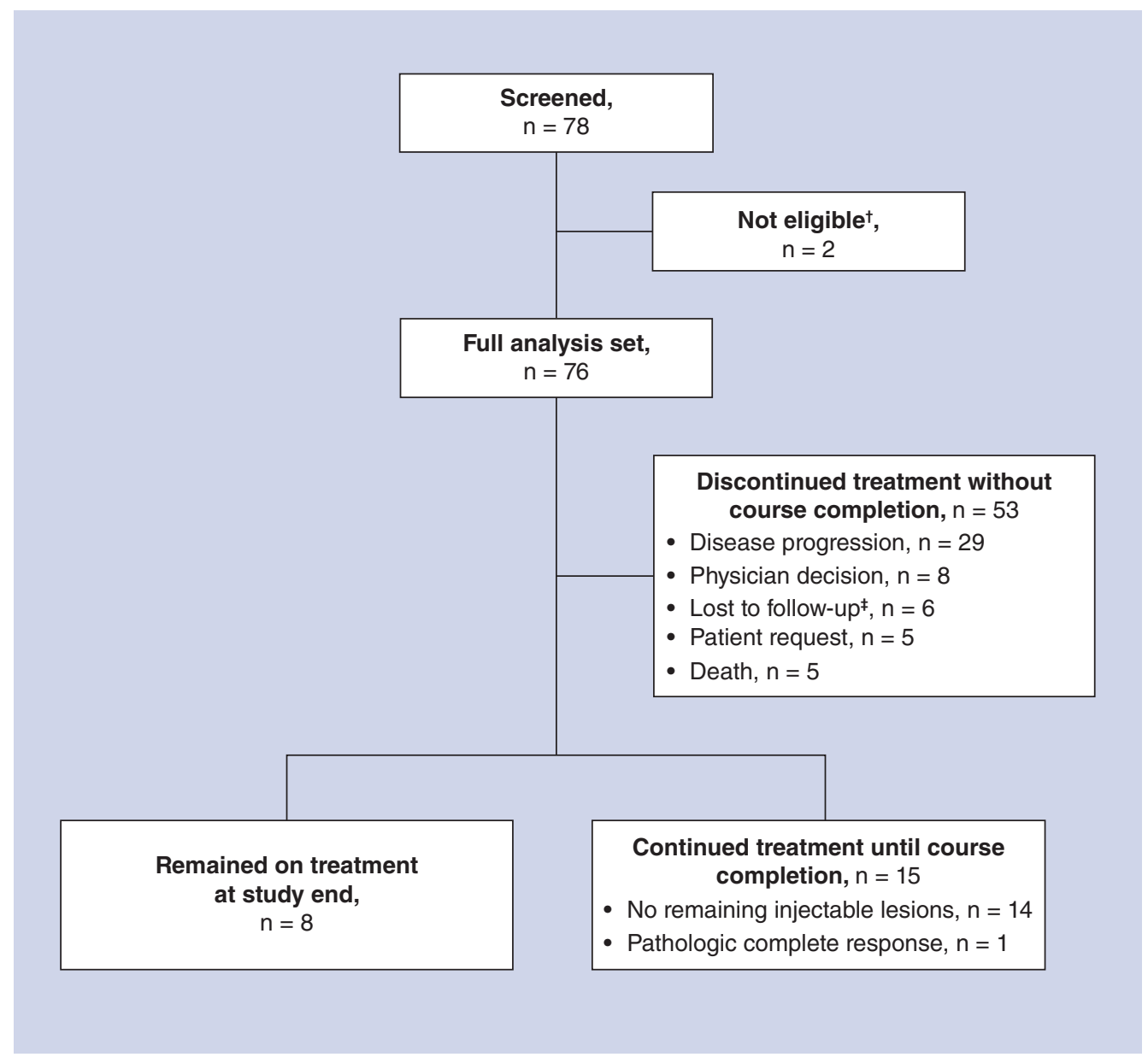

Figure 2. Disposition of patients.

$\dagger$ Participated in talimogene laherparepvec clinical trial or expanded access program.

$\ddagger$ Did not return to the clinic, left the clinic to initiate or continue treatment at an outside institution, were no longer covered byinsurance, or were receiving other therapies at time of discontinuation of talimogene laherparepvec.

\begin{tabular}{|c|c|c|c|c|c|c|c|}
\hline \multirow[t]{2}{*}{ T-VEC exposure } & \multirow[t]{2}{*}{ n (\%) } & $\begin{array}{l}\text { Volume }(\mathrm{mL}) \text { per } \\
\text { visit at } 10^{6} \text { : } \\
\text { visit } 1\end{array}$ & $\begin{array}{l}\text { Volume }(\mathrm{mL}) \text { per } \\
\text { visit at } 10^{8}: \\
\text { visits } 2-10\end{array}$ & $\begin{array}{l}\text { Volume }(\mathrm{mL}) \text { per } \\
\text { visit at } 10^{8}: \\
\text { visits } \geq 11\end{array}$ & $\begin{array}{l}\text { Cumulative } \\
\text { volume }(\mathrm{mL}) \text { : } \\
\text { all visits }\end{array}$ & $\begin{array}{l}\text { Treatment } \\
\text { duration } \\
\text { (months) }\end{array}$ & $\begin{array}{l}\text { Number of } \\
\text { visits }\end{array}$ \\
\hline & & $\begin{array}{l}\text { Median (min, } \\
\text { max) }\end{array}$ & Median & Median & Median & Median & Median \\
\hline All patients & $76(100)$ & $2.0(0.2,4.0)$ & 2.0 & 1.3 & 12.0 & 2.8 & 6.0 \\
\hline - Successfully completed treatment ${ }^{\dagger}$ & $15(19.7)$ & $1.3(0.4,4.0)$ & 1.3 & 0.8 & 11.0 & 3.0 & 7.0 \\
\hline - Discontinued T-VEC $\ddagger$ & $53(69.7)$ & $3.0(0.2,4.0)$ & 2.8 & 2.7 & 12.0 & 2.6 & 5.0 \\
\hline - Continued T-VEC & $8(10.5)$ & $1.3(0.5,4.0)$ & 1.8 & 1.8 & 22.3 & $8.0^{\S}$ & 12.0 \\
\hline \multicolumn{8}{|c|}{$\begin{array}{l}\dagger \text { †No injectable lesions remaining or pathologic complete response. } \\
\ddagger \text { Disease progression, death, physician decision, patient request or lost to follow-up. } \\
\text { § Two patients received two treatment cycles with T-VEC each, and each treatment cycle with T-VEC is accounted for in the calculation. } \\
\text { T-VEC: Talimogene laherparepvec. }\end{array}$} \\
\hline
\end{tabular}

\section{Other clinical outcomes \& treatment patterns}

Overall, 15 patients (19.7\%) continued treatment with T-VEC until completion, which was defined as either no further injectable lesions or a documented pathologic complete response (CR). Distant metastases were diagnosed after the first dose of T-VEC in two patients (2.6\%). The median duration of T-VEC therapy was 3.0 months (range, 1.6-9.0 months) in patients treated to course completion $(n=15)$. Table 2 gives the median T-VEC 


\begin{tabular}{|c|c|c|c|c|c|}
\hline $\begin{array}{l}\text { Received another therapy prior to } \\
\text { T-VEC, } \mathrm{n}(\%)^{\ddagger}\end{array}$ & $\begin{array}{l}\text { Total } \\
(\mathrm{N}=76)\end{array}$ & $\begin{array}{l}\text { Received another therapy concurrent } \\
\text { with T-VEC, } \mathrm{n}(\%)\end{array}$ & $\begin{array}{l}\text { Total } \\
(\mathrm{N}=76)\end{array}$ & $\begin{array}{l}\text { Received another therapy after T-VEC, } n \\
\text { (\%) }\end{array}$ & $\begin{array}{l}\text { Total } \\
(\mathrm{N}=76)\end{array}$ \\
\hline Ipilimumab & $19(25.0)$ & T-VEC + pembrolizumab & $8(10.5)$ & Pembrolizumab & $8(10.5)$ \\
\hline Clinical trial for melanoma & $15(19.7)$ & T-VEC + radiation & $4(5.3)$ & Radiation & $3(3.9)$ \\
\hline Pembrolizumab & $14(18.4)$ & T-VEC + nivolumab & $3(3.9)$ & Dabrafenib & $2(2.6)$ \\
\hline Radiation & $14(18.4)$ & T-VEC + ipilimumab + nivolumab & $2(2.6)$ & Ipilimumab & $2(2.6)$ \\
\hline Melphalan $\S$ & $11(14.5)$ & T-VEC + ipilimumab & $1(1.3)$ & Ipilimumab + nivolumab & $2(2.6)$ \\
\hline Interferon $\mathbb{I}$ & $10(13.2)$ & T-VEC + vemurafenib & $1(1.3)$ & Trametinib & $2(2.6)$ \\
\hline Nivolumab & $6(7.9)$ & T-VEC + vemurafenib + dabrafenib & $1(1.3)$ & Vemurafenib + dabrafenib & $2(2.6)$ \\
\hline Interleukin-2\# & $5(6.6)$ & & & Clinical trial for melanoma & $1(1.3)$ \\
\hline Temozolomide & $4(5.3)$ & & & Cisplatin + vinblastine & $1(1.3)$ \\
\hline Ipilimumab + nivolumab & $3(3.9)$ & & & Cobimetinib + vemurafenib & $1(1.3)$ \\
\hline Dabrafenib & $2(2.6)$ & & & Dabrafenib + trametinib & $1(1.3)$ \\
\hline Dabrafenib + trametinib & $2(2.6)$ & & & Larotrectinib & $1(1.3)$ \\
\hline GM-CSF & $2(2.6)$ & & & Vemurafenib & $1(1.3)$ \\
\hline Carboplatin + docetaxel & $1(1.3)$ & & & Vemurafenib + cobimetinib & $1(1.3)$ \\
\hline Carboplatin + nab-paclitaxel & $1(1.3)$ & & & & \\
\hline Carboplatin + paclitaxel & $1(1.3)$ & & & & \\
\hline Cisplatin + dacarbazine + vinblastine & $1(1.3)$ & & & & \\
\hline Cisplatin + vinblastine + temozolomide & $1(1.3)$ & & & & \\
\hline Dacarbazine & $1(1.3)$ & & & & \\
\hline Peginterferon alpha-2b & $1(1.3)$ & & & & \\
\hline Topical imiquimod & $1(1.3)$ & & & & \\
\hline Vemurafenib & $1(1.3)$ & & & & \\
\hline Vemurafenib + dabrafenib & $1(1.3)$ & & & & \\
\hline Vemurafenib + temozolomide & $1(1.3)$ & & & & \\
\hline Treated with T-VEC only, n (\%) & $\begin{array}{l}\text { Total } \\
(\mathrm{N}=76)\end{array}$ & & & & \\
\hline T-VEC ${ }^{\dagger \dagger}$ & $17(22.4)$ & & & & \\
\hline \multicolumn{6}{|c|}{$\begin{array}{l}\text { †Patients may have received more than one other type of therapy before, concurrent with, or after T-VE } \\
\text { ¥The subcategories within the melphalan, interferon and IL-2 categories are not mutually exclusive. Sub } \\
\S \text { Melphalan includes melphalan, melphalan + actinomycin-d, right leg hyperthermic isolated limb perfusic } \\
\text { IInterferon includes adjuvant interferon, adjuvant interferon (systemic), high-dose interferon, interferon } \\
\text { \#Interleukin-2 includes intralesional injection with Interleukin-2 and high-dose Interleukin-2. } \\
\dagger \dagger \text { No record of patients receiving other antineoplastic treatment before, concurrent with or after T-VEC. } \\
\text { GM-CSF: Granulocyte macrophage colony-stimulating factor; T-VEC: Talimogene laherparepvec. }\end{array}$} \\
\hline
\end{tabular}

exposure and Supplementary Table 1 gives the mean exposure.

Fifty-three patients (69.7\% of all 76 patients) discontinued T-VEC treatment before a CR was demonstrated. Among these 53 patients, reasons for discontinuing treatment without achieving a CR were disease progression $(\mathrm{n}=29 ; 54.7 \%)$, physician decision $(\mathrm{n}=8 ; 15.1 \%)$, loss to follow-up $(\mathrm{n}=6 ; 11.3 \%)$, patient request $(\mathrm{n}=5 ; 9.4 \%)$ or death $(n=5 ; 9.4 \%)$.

In patients who did not discontinue treatment with T-VEC, median duration of therapy was 8.0 months for patients who continued on T-VEC therapy at the end of data collection $(n=8)$ and 2.6 months for patients who had discontinued T-VEC prior to the end of data collection $(n=53)$.

Seventeen patients (22.4\%) received T-VEC as the sole melanoma therapy with no other recorded antineoplastic treatment before, concurrent with or after treatment with T-VEC.

\section{Clinical outcomes \& treatment patterns with use of checkpoint inhibitors}

Of 76 patients treated with T-VEC in the overall study population, 33 (43.4\%) had received pembrolizumab, nivolumab and/or ipilimumab (i.e., a checkpoint inhibitor) before or concurrent with T-VEC. A greater number of patients received checkpoint inhibitors prior to T-VEC $(n=21)$ compared to concurrently with T-VEC $(n=12)$. As shown in Table 3, in patients who received checkpoint inhibitors prior to T-VEC, prior treatment with 


\begin{tabular}{|c|c|c|c|c|}
\hline \multirow[t]{2}{*}{ Outcomes and treatment sequence for T-VEC and CPI } & \multicolumn{3}{|c|}{ Discontinued treatment with T-VEC } & \multirow{2}{*}{$\begin{array}{l}\text { Completed treatment } \\
\text { with T-VEC }(n=3)^{\dagger}\end{array}$} \\
\hline & $\begin{array}{l}\text { Disease progression } \\
(n=21)\end{array}$ & $\begin{array}{l}\text { Physician decision to } \\
\text { discontinue }(n=6)\end{array}$ & $\begin{array}{l}\text { Patient request to } \\
\text { discontinue }(n=2)\end{array}$ & \\
\hline \multicolumn{5}{|l|}{ T-VEC prior to or after CPI: } \\
\hline$-\mathrm{T}-\mathrm{VEC} \rightarrow \mathrm{CPI}$ & $8(38.1)$ & $0(0.0)$ & $0(0.0)$ & $0(0.0)$ \\
\hline$-\mathrm{CPI} \rightarrow \mathrm{T}$-VEC & $10(47.6)$ & $4(66.7)$ & $1(50.0)$ & $3(100.0)$ \\
\hline \multicolumn{5}{|l|}{ T-VEC concurrent with CPI: } \\
\hline$-\mathrm{T}-\mathrm{VEC}+\mathrm{CPI} \rightarrow \mathrm{CPI}$ & $1(4.8)$ & $1(16.7)$ & $0(0.0)$ & $0(0.0)$ \\
\hline$-\mathrm{CPI} \rightarrow \mathrm{T}-\mathrm{VEC}+\mathrm{CPI}$ & $2(9.6)$ & $1(16.7)$ & $1(50.0)$ & $0(0.0)$ \\
\hline
\end{tabular}

$\dagger$ Patients who discontinued due to no injectable lesions or due to pathologic complete response.

CPI: Checkpoint inhibitor; T-VEC: Talimogene laherparepvec.

ipilimumab $(\mathrm{n}=19)$ or pembrolizumab $(\mathrm{n}=14)$ was more common than treatment with nivolumab $(\mathrm{n}=6)$ or ipilimumab + nivolumab $(n=3)$. In patients who received checkpoint inhibitors concurrent with T-VEC, concurrent treatment with pembrolizumab was more common $(n=8)$ than treatment with nivolumab $(n=3)$, ipilimumab + nivolumab $(\mathrm{n}=2)$ or ipilimumab $(\mathrm{n}=1)$.

Selected clinical outcomes for patients who received T-VEC and checkpoint inhibitors in various treatment sequences are shown in Table 4. A similar total number of patients discontinued T-VEC due to disease progression ( $\mathrm{n}=10,8$, respectively) whether they received checkpoint inhibitors prior to or after T-VEC monotherapy (of $\mathrm{n}=21,8$ in each treatment group, respectively). Of the 21 patients who received a checkpoint inhibitor prior to T-VEC monotherapy, three patients completed treatment with either no injectable lesions remaining or a pathologic CR. All of the eight patients who received a checkpoint inhibitor after T-VEC monotherapy experienced disease progression. Of the 12 patients who received a checkpoint inhibitor concurrent with T-VEC, two remained on T-VEC at the end of data collection and three experienced disease progression.

\section{Safety}

In this study, specific AEs of interest were systematically collected in three categories: immune-mediated events, injection site complications and herpetic events. AEs of interest were reported for 21 of 76 patients $(27.6 \%$; Table 5). Flu-like symptoms were most common $(n=8,10.5 \%)$, followed by lesion ulceration $(n=4,5.3 \%)$. No herpetic lesions or infections were reported.

Among the 21 patients who received a checkpoint inhibitor before T-VEC, AEs of interest were reported in seven patients. Among the 12 patients who received a checkpoint inhibitor concurrent with T-VEC, AEs of interest were reported in six patients. The most common AEs of interest in both groups were immune-mediated events $(\mathrm{n}=3$ and 6), which included flu-like symptoms (fever, chills and rigor; $\mathrm{n}=2$ and 5), and injection site complications ( $\mathrm{n}=5$ and 2), for patients who received a checkpoint inhibitor before or concurrent with T-VEC, respectively. No herpetic lesions or infections were reported in the patients who received a checkpoint inhibitor before or concurrent with T-VEC.

\section{Discussion}

T-VEC was well tolerated and showed clinical utility in this real-world cohort of US patients with unresectable regional and distant metastatic melanoma. 19.7\% $(n=15)$ of patients completed treatment with T-VEC with no remaining injectable lesions or pathologic CR. No herpetic lesions or infections were recorded in this group of patients who received T-VEC in a real-world practice situation. A substantial proportion of patients received checkpoint inhibitors before, after or concurrent with T-VEC.

Overall, efficacy and safety findings in this real-world data study complement the results of the randomized, open-label, Phase III OPTiM trial comparing intralesional T-VEC with subcutaneous GM-CSF in patients with unresected stage IIIB-IV melanoma [7]. In OPTiM, the overall response rate (ORR) for the T-VEC and GM-CSF arms was $26.4 \%$ versus $5.7 \%$, and the CR rate was $10.8 \%$ versus less than $1 \%$. In the COSMUS- 1 study, $19.7 \%$ $(\mathrm{n}=15)$ of patients completed treatment with T-VEC with no remaining injectable lesions or pathologic CR. In OPTiM, there was a trend toward improved OS with T-VEC (23.3 vs 18.9 months with GM-CSF). Whereas, in the COSMUS-1 study, OS rates at 1 year were $76.7 \%$ in patients with stage IIIB-IVM1a and $64.6 \%$ in patients 


\begin{tabular}{|c|c|}
\hline & Total $(n=76)$ \\
\hline Total, n (\%) & $21(27.6)$ \\
\hline \multicolumn{2}{|l|}{ Immune-mediated events, n (\%): } \\
\hline - Any immune-mediated event & $12(15.8)$ \\
\hline - Flu-like symptoms (fever, chills, rigors) & $8(10.5)$ \\
\hline - Fatigue & $2(2.6)$ \\
\hline - Joint aches & $1(1.3)$ \\
\hline - Muscle aches & $1(1.3)$ \\
\hline - Rash & $1(1.3)$ \\
\hline - Vomiting & $1(1.3)$ \\
\hline - Severe inflammation involving the eye & $1(1.3)$ \\
\hline - Severe erythema involving the eye & $1(1.3)$ \\
\hline \multicolumn{2}{|l|}{ Injection site complications, n (\%): } \\
\hline - Any injection site complication & $12(15.8)$ \\
\hline - Ulceration of tumor & $4(5.3)$ \\
\hline - Injection site pain & $2(2.6)$ \\
\hline - Cellulitis & $2(2.6)$ \\
\hline - Necrosis & $1(1.3)$ \\
\hline - Arm swelling & $1(1.3)$ \\
\hline - Erythema & $1(1.3)$ \\
\hline - Purulent drainage & $1(1.3)$ \\
\hline - Severe inflammation and erythema around site of injection & $1(1.3)$ \\
\hline
\end{tabular}

with stage IVM1b-IVM1c melanoma. Key AEs of interest in COSMUS-1 were also similar to those in OPTiM [7], with the most common AEs of interest being flu-like symptoms and lesion ulceration.

Compared with the overall median duration of treatment in OPTiM (5.8 months), median duration of therapy was shorter (3.0 months) for COSMUS-1 patients who completed treatment with T-VEC with either no further injectable lesions or a documented pathologic CR. The median duration of therapy was the longest ( 8.0 months) for COSMUS-1 patients who continued on T-VEC therapy at the end of data collection; these patients were presumably continuing to derive clinical benefit. For COSMUS-1 patients who discontinued T-VEC prior to the end of data collection, the median duration of therapy was shorter (2.6 months). Overall median duration of treatment in OPTiM (5.8 months) was likely influenced by protocol specifications for minimum of 24 weeks (6 months) of treatment [7].

The present study was limited in its ability to delineate reasons for T-VEC discontinuation, such as the rationales behind physicians' decisions to discontinue, as this level of detail was not collected in the dataset. In addition, direct quantitative comparisons cannot be made between real-world data and data from randomized, controlled clinical trials; the populations differ and some outcomes, such as pseudoprogression or CR, are not recorded by clinicians in medical charts in the same way that they are captured in clinical trials. CR in trials is generally confirmed by pathologic reports, which may not be systematically available in a medical chart review study such as COSMUS-1. Therefore, COSMUS-1 also considered patients with no remaining injectable lesions as having completed treatment.

In addition to the results presented here, other reports of T-VEC in clinical practice have provided a body of evidence about the use of T-VEC as monotherapy and in combination with checkpoint inhibitors [13-17]. In a single-center US study of medical records from 27 patients with advanced melanoma who received T-VEC in 2015-2018, AEs were mostly mild and the disease control rate was 78.3\% [14]. A multicenter, retrospective German study of 27 patients with unresectable earlier stage IIIB-IVM1a melanoma who received T-VEC reported that T-VEC was the initial systemic therapy in $63 \%$ of patients, no new safety signals were reported and three patients completed T-VEC treatment with no remaining injectable lesions [15]. A large multicenter US study of 121 patients who received T-VEC in 2015-2018 found that 42.5\% of patients received the injections as first-line 
therapy, side effects were mild and 37\% of the complete responders had no evidence of disease at last follow-up [16]. A single-site study of 26 T-VEC-treated patients from the Netherlands recently presented similara similar safety profile as previously reported and a best ORR of $88.5 \%$ [22]. Of these studies, three reported ORR and CR, which ranged from approximately $57-89 \%$ and $39-62 \%$, respectively $[14,16,22]$. In COSMUS-1, 55.3\% of patients had earlier stage IIIB-IVM1a melanoma, whereas two of these recent studies included only earlier stage IIIB-IVM1a patients $[14,22]$ and one study included a majority $(67 \%)$ of earlier stage IIIB-IVM1a patients. The lower proportion of patients with earlier stage IIIB-IVM1a melanoma in COSMUS-1 may be a factor in the differences in response rate seen across these studies.

A growing consensus suggests that the next wave of melanoma treatment will include combination approaches [1720]. In COSMUS-1, $43.4 \%$ of patients received a checkpoint inhibitor (pembrolizumab, nivolumab and/or ipilimumab) prior to or concurrent with T-VEC. Patients were more likely to have received checkpoint inhibitor therapy (most notably ipilimumab or pembrolizumab) prior to T-VEC as opposed to concurrent with T-VEC. It is worth noting that in the COSMUS-1 cohort, one patient demonstrated a pathologic CR, and this patient had received treatment with a checkpoint inhibitor concurrent with T-VEC. Locally administered immunotherapy, including in combination with checkpoint inhibitors, will likely be an important option when individualizing melanoma therapies [21].

\section{Conclusion}

In this real-world cohort, treatment with T-VEC was documented in locoregionally aggressive and distantly metastatic melanoma, both as monotherapy and in combination with treatments such as checkpoint inhibitors. Twenty percent of patients discontinued T-VEC treatment because of no remaining injectable lesions or achievement of pathologic CR. Overall, T-VEC was well tolerated and clinically efficacious with outcomes comparable to those previously reported in the OPTiM trial and in other retrospective reviews.

Supplementary data

To view the supplementary data that accompany this paper please visit the journal website at: www.futuremedicine.com/doi/full/ 10.2217/mmt-2019-0012

\section{Author contributions}

MC Perez, JS Zager, T Amatruda, R Conry, C Ariyan, A Desai, JM Kirkwood, S Treichel, D Cohan and L Raskin were responsible for study conception and design; The authors MC Perez, JS Zager, T Amatruda, R Conry, C Ariyan, A Desai and JM Kirkwood were responsible for acquisition of data; the authors MC Perez, JS Zager, T Amatruda, R Conry, C Ariyan, A Desai, JM Kirkwood, $S$ Treichel, D Cohan and L Raskin were responsible for data analysis, and drafting and revision of the manuscript.

Financial \& competing interests disclosure

This study was funded by Amgen Inc. MC Perez received research funding from Amgen Inc for this project. JS Zager has engaged in consultancies from Amgen Inc, Philogen, Merck, Castle Biosciences; honoraria from Amgen Inc, Array BioPharma; stock options from Delcath Systems; expert testimony for McGowan Hood; grants or patents received or pending from Delcath Systems. T Amatruda is involved in editing and reviewing of clinical trial reports from Amgen Inc. R Conry has recieved honoraria for speaker bureau from Amgen Inc, Merck, BMS, Novartis, Array, Regeneron, Sanofi. C Ariyan has stock ownership or options from Pfizer. A Desai has recieved travel funding from Amgen Inc. JM Kirkwood: grants from Merck, Prometheus, Immunocore LLC; personal fees from Immunocore LLC, Array Biopharma, BMS, Novartis, Roche. S Treichel is an employee of Amgen Inc; owns stock or options from Amgen Inc. D Cohan is an employee of Amgen Inc; owns stock or options from Amgen Inc. L Raskin is an employee of Amgen Inc; owns stock or options from Amgen Inc.

The authors have no other relevant affiliations or financial involvement with any organization or entity with a financial interest in or financial conflict with the subject matter or materials discussed in the manuscript apart from those disclosed.

Writing assistance was provided by Caley-Reidenbach Consulting, LLP, which was funded by Amgen Inc.

Previous presentation

Results of this study were presented at the Fifteenth International Congress of the Society for Melanoma Research (24-27 October 2018, Manchester, England). 


\section{Ethical conduct of research}

The authors state that they have obtained appropriate institutional review board approval or have followed the principles outlined in the Declaration of Helsinki for all human or animal experimental investigations. In addition, for investigations involving human subjects, informed consent has been obtained from the participants involved.

\section{Open access}

This work is licensed under the Attribution-NonCommercial-NoDerivatives 4.0 Unported License. To view a copy of this license, visit http://creativecommons.org/licenses/by-nc-nd/4.0/

\section{References}

Papers of special note have been highlighted as: • of interest; $\bullet \bullet$ of considerable interest

1. Bhandaru M, Rotte A. Monoclonal antibodies for the treatment of melanoma: present and future strategies. Methods Mol. Biol. 1904, 83-108 (2019).

2. Lugowska I, Teterycz P, Rutkowski P et al. Immunotherapy of melanoma. Contemp. Oncol. (Pozn). 22(1A), 61-67 (2018).

3. Gershenwald JE, Scolyer RA, Hess KR et al. Melanoma staging: evidence-based changes in the American Joint Committee on Cancer eighth edition cancer staging manual. CA Cancer J. Clin. 67(6), 472-492 (2017).

4. Luu C, Khushalani NI, Zager JS. Intralesional and systemic immunotherapy for metastatic melanoma. Expert Opin. Biol. Ther. 16(12), 1491-1499 (2016).

5. Miura JT, Zager JS. Intralesional therapy as a treatment for locoregionally metastatic melanoma. Expert Rev. Anticancer Ther. 18(4), 399-408 (2018).

6. Ledford H. Cancer-fighting viruses win approval. Nature 526(7575), 622-623 (2015).

7. Andtbacka RH, Kaufman HL, Collichio F et al. Talimogene laherparepvec improves durable response rate in patients with advanced melanoma. J. Clin. Oncol. 33(25), 2780-2788 (2015).

-• Reports the primary results of the OPTiM randomized open-label Phase III clinical trial that provided the principal registrational data for talimogene laherparepvec (T-VEC).

8. $\quad$ IMLYGIC ${ }^{\circledR}$. Summary of Product Characteristics. Amgen Ltd, Cambridge, UK (2018).

9. Dummer R, Hoeller C, Gruter IP, Michielin O. Combining talimogene laherparepvec with immunotherapies in melanoma and other solid tumors. Cancer Immunol. Immunother. 66(6), 683-695 (2017).

10. Goldsmith K, Chen W, Johnson DC, Hendricks RL. Infected cell protein (ICP) 47 enhances herpes simplex virus neurovirulence by blocking the CD8 ${ }^{+}$T cell response. J. Exp. Med. 187(3), 341-348 (1998).

11. Poppers J, Mulvey M, Khoo D, Mohr I. Inhibition of PKR activation by the proline-rich RNA binding domain of the herpes simplex virus type 1 Us11 protein. J. Virol. 74(23), 11215-11221 (2000).

12. Balch CM, Gershenwald JE, Soong SJ et al. Final version of 2009 AJCC melanoma staging and classification. J. Clin. Oncol. 27(36), 6199-6206 (2009).

13. Sun L, Funchain P, Song JM et al. Talimogene laherparepvec combined with anti-PD-1 based immunotherapy for unresectable stage III-IV melanoma: a case series. J. Immunother. Cancer. 6(1), 36 (2018).

- A case series from the Cleveland Clinic of ten patients with stage IIIC-IVM1b melanoma who received T-VEC plus checkpoint inhibitor therapy.

14. Perez MC, Miura JT, Naqvi SMH et al. Talimogene laherparepvec (TVEC) for the treatment of advanced melanoma: a single-institution experience. Ann. Surg. Oncol. 25(13), 3960-3965 (2018).

- A study from the Moffitt Cancer Center of medical records from 27 patients with advanced melanoma who received T-VEC between 2015 and 2018.

15. Mohr P, Haferkamp S, Pinter A et al. Real-world use of talimogene laherparepvec in German patients with stage IIIB to IVM1a melanoma: a retrospective chart review and physician survey. Adv. Ther. 36(1), 101-117 (2019).

- A multicenter, retrospective German study of 27 patients with unresectable stage IIIB-IVM1a melanoma who received T-VEC in clinical practice.

16. Louie RJ, Perez MC, Jajja MR et al. Real world outcomes of talimogene laherparepvec therapy: a multi-institutional experience. J. Am. Coll. Surg. doi: 10.1016/j.jamcollsurg.2018.12.027 (2019) (Epub ahead of print).

- A multicenter US study of 121 patients who received T-VEC in routine practice between 2015 and 2018.

17. Cavalcante L, Chowdhary A, Sosman JA, Chandra S. Combining tumor vaccination and oncolytic viral approaches with checkpoint inhibitors: rationale, pre-clinical experience, and current clinical trials in malignant melanoma. Am. J. Clin. Dermatol. 19(5), 657-670 (2018).

18. Lawler SE, Speranza MC, Cho CF, Chiocca EA. Oncolytic viruses in cancer treatment: a review. JAMA Oncol. 3(6), 841-849 (2017). 
19. Ascierto PA, Puzanov I, Agarwala SS et al. Perspectives in melanoma: meeting report from the Melanoma Bridge ( 30 November-2 December, 2017, Naples, Italy). J. Transl. Med. 16(1), 207 (2018).

20. Collins JM, Redman JM, Gulley JL. Combining vaccines and immune checkpoint inhibitors to prime, expand, and facilitate effective tumor immunotherapy. Expert Rev. Vaccines. 17(8), 697-705 (2018).

21. Aznar MA, Tinari N, Rullan AJ, Sanchez-Paulete AR, Rodriguez-Ruiz ME, Melero I. Intratumoral delivery of immunotherapy: act locally, think globally. J. Immunol. 198(1), 31-39 (2017).

22. Franke V, Berger DMS, Klop WMC et al. High response rates for T-VEC in early metastatic melanoma (stage IIIB/C-IVM1a). Int. J. Cancer doi: 10.1002/ijc.32172 (2019) (Epub ahead of print). 